\title{
Suspicion de la résistance aux benzimidazoles chez les strongles gastro- intestinaux du caprin à Lubumbashi, R.D. Congo
}

\author{
E.V. OKOMBE* et S.E.W. PONGOMBO
}

\author{
Service de Pharmacologie, Toxicologie et Thérapeutique, Faculté de Médecine Vétérinaire, \\ Université de Lubumbashi, B.P. 1825 Lubumbashi, République Démocratique du Congo. \\ *Auteur correspondant, E-mail : vokombe@yahoo.fr; Tél : (+243) 818152587
}

\section{RESUME}

Cent vingt-quatre caprins sélectionnés dans deux fermes de la région de Lubumbashi en R.D. Congo, ont été examinés en utilisant le test de réduction de l'excrétion fécale des œufs de strongles dans les fèces afin de détecter une résistance éventuelle aux anthelminthiques utilisés. Dans les deux fermes, il a été suspecté une résistance de strongles digestifs face aux benzimidazoles (29,9 à 48,9\% de réduction) comparativement à l'ivermectine (95,6 à 96,7\% de réduction). Cette étude a permis de suspecter des cas de résistance aux benzimidazoles chez les nématodes gastro-intestinaux des petits ruminants à Lubumbashi en R.D. Congo. Des recherches plus poussées, soutenues, devraient être poursuivies afin de confirmer cette résistance des nématodes aux anthelminthiques dans la région et de déterminer les espèces de parasites mises en cause.

(C) 2013 International Formulae Group. All rights reserved.

Mots clés : Résistance, benzimidazoles, strongles gastro-intestinaux, caprin, Lubumbashi.

\section{INTRODUCTION}

À Lubumbashi, l'élevage de la chèvre est une activité importante, qui permet aux éleveurs d'assurer un revenu complémentaire, seul moyen, pour bien des familles, de contribuer à la réalisation des projets (construction d'une maison, achat d'un lopin de terre) ou de faire face à certaines dépenses (frais liés à une maladie, scolarisation, mariage) (Okombe et al., 2013).

Cependant, cet élevage rencontre des difficultés, liées notamment à la conduite du troupeau et à la gestion des maladies telles que les verminoses gastro-intestinales.

Les infestations par les nématodes parasites du tube digestif représentent une des dominantes pathologiques des animaux élevés à l'herbe (Demeler et al., 2009 ; Sutherland and Leathwick, 2010), mode d'exploitation utilisé à Lubumbashi. Elles constituent ainsi une cause importante de mortalité et sont responsables de la baisse de production (Coles et al., 2006). Ces strongyloses sont dues à des vers ronds présents à différents niveaux du tube digestif de l'animal hôte, occasionnant des dommages d'intensité variable.

Pour lutter contre ces parasites indésirables, les éleveurs ont eu recours, le plus souvent, à l'utilisation de substances chimiques : les anthelminthiques. La gamme des anthelminthiques disponibles sur le marché local est limitée aux benzimidazoles et aux lactones macrocycliques. Malheureusement, leur utilisation répétée à 
long terme permet aux parasites de s'adapter en développant une résistance irréversible à la majorité des familles des molécules (Besier and Love, 2003).

La résistance correspond au développement de la capacité d'individus à tolérer des doses de toxique qui seraient mortelles pour la majorité des individus d'une population normale de la même espèce (Fao, 2004 ; Levecke et al., 2012). Il s'agit d'une préadaptation par mutation dont le déterminisme est génétique et la transmission héréditaire. Elle peut concerner une seule molécule, on parle alors de résistance simple, ou deux, voir plusieurs molécules apparentées ou non : c'est la résistance multiple.

Ce phénomène s'étend de plus en plus à l'ensemble des régions du monde (Conder and Campbell, 1995) et touche presque toutes les espèces de strongles (Chartier et al., 1997). C'est un sujet aujourd'hui préoccupant qui prend de plus en plus d'ampleur (Chartier et al., 1996).

Les résistances les plus importantes se rapportent aux ovins avec comme principale molécule incriminée celle des benzimidazoles, à cause probablement du fait qu'ils soient les premiers anthelminthiques à large spectre d'action contre les strongles digestifs et les moins chers, ce qui peut influencer les éleveurs dans leur utilisation exclusive et abusive (Suarez et al., 2007).

Les pays tropicaux ne sont pas épargnés (Van Wyk et al., 1997). Dans bon nombre d'entre eux (Kenya, Zimbabwe), des résistances aux trois principales familles d'anthelminthiques ont déjà été répertoriées (Mukaratirwa et al., 1997. Waller, 1997 ; Waruiru et al., 1998b) et concernent fréquemment Haemonchus contortus. $\mathrm{Au}$ Kenya, plus de la moitié des exploitations présentent une résistance à au moins un groupe d'anthelminthiques (Waller, 1997). L'étude de Waruiri et al. (1998a) a mis en évidence une résistance simultanée d'Haemonchus contortus aux benzimidazoles, lévamisole et rafoxanide ainsi que la résistance de Trichostrongylus colubriformis et Oesophagostomum sp. au lévamisole (Waruiru et al., 1998b).
Par ailleurs, une étude (Ba, 1998) a permis la découverte de cas de résistance aux benzimidazoles dans des troupeaux ovins d'Afrique occidentale (en Gambie et au Sénégal), épargnés jusqu'alors.

En Afrique du Sud, le développement des résistances d'Haemonchus contortus à tous les anthelminthiques disponibles laisse peu d'espoir aux éleveurs (Van Wyk et al., 1999). Plus de $90 \%$ des élevages ovins sont confrontés au problème et certains ont même dû être éliminés pour des raisons économiques. Les premières populations d'Haemonchus contortus résistantes à l'ivermectine y ont été décrites pour la première fois en 1985 (Van Wyk et al., 1999).

Face aux plaintes des éleveurs sur la persistance des symptômes après administration des anthelminthiques, nous avons pensé qu'il était opportun d'aborder la question et d'orienter nos recherches vers le phénomène de résistance.

Le but de cette étude est de vérifier, par le test de réduction d'excrétion fécale des œufs (Faecal Egg Count Reduction Test), l'efficacité de trois anthelminthiques à usage courant (ivermectine, albendazole et oxfendazole) et l'éventuelle apparition d'une résistance chez les parasites des caprins élevés sur pâturage à Lubumbashi.

\section{MATERIEL ET METHODES}

L'étude a été réalisée en février 2012 et en mars 2013 dans deux fermes situées en périphérie de la ville de Lubumbashi sélectionnées pour leur parasitisme moyen, supérieur à 350 œufs par gramme et dans lesquelles la coprologie a révélé, après traitement aux benzimidazoles (avec une répétition après deux semaines), la persistance élevée des œufs de nématodes dans les fèces. Ces élevages ont été sélectionnés parmi ceux effectuant une vermifugation régulière de leurs animaux.

Dans chaque élevage, il a été effectué une pré-enquête consistant à déterminer le degré d'infestation des animaux.

L'étude a porté sur 124 caprins de deux sexes, âgés d'au moins trois mois, naturellement infestés par divers helminthes 
gastro-intestinaux et qui n'avaient reçu aucun traitement anthelminthique depuis 3 mois.

En 2012, nous avons travaillé sur 64 animaux répartis en 4 lots de 16 animaux et en 2013, nous avons travaillé sur 60 animaux répartis en 4 lots de 15 caprins chacun. Les animaux ont été laissés pâturer ensemble et les lots n'ont pas été séparés physiquement pendant le temps de l'étude.

Les traitements suivants ont été administrés aux différents lots :

- le lot 1 a été le lot témoin non traité ;

- le lot 2 a été traité à l'albendazole à la dose de $5 \mathrm{mg} / \mathrm{kg}$ (Valbazen®1,9\%);

- le lot 3 a été traité à l'oxfendazole à la dose de $11,3 \mathrm{mg} / \mathrm{kg}$ (Dolthene®);

- le lot 4 a été traité à l'ivermectine à la dose de $0,2 \mathrm{mg} / \mathrm{kg} \mathrm{kg}$ (Ivomec®).

La récolte des crottes a été faite à l'aide des doigts préalablement protégés par des gants stériles en plastique, dans le rectum de chaque sujet d'étude. Les crottins ont été mis dans des sachets en plastiques numérotés. L'analyse coprologique a été réalisée au laboratoire de parasitologie de la faculté de Médecine Vétérinaire de Lubumbashi, selon une technique classique de flottaison en solution saline (Urquhart et al., 1996) d'une densité de 1,2.

Pour la quantification des œufs par gramme de matières fécales (Opg), nous avons utilisé la technique de McMaster (Thienpont et al., 1979), à deux chambres, à un seuil minimal de 50 Opg.

Pour la détermination de la résistance aux anthelminthiques, nous avons utilisé le Test de réduction de l'excrétion des œufs dans les matières fécales, «FECR » (Faecal Egg Count Reduction Test).

Ce test est basé sur la réduction du nombre d'œufs dans les fèces après traitement (Boulkaboul et al., 2006).

Ce taux a été calculé selon la méthode de Dash (1988) qui considère les Opg moyens avant et après les traitements suivant la formule :

$\operatorname{FECR}(\%)=[1-(\mathrm{T} 1 / \mathrm{T} 2)(\mathrm{C} 2 / \mathrm{C} 1)] * 100$

Avec FECR \% = Taux de réduction fécale;

$\mathrm{T} 1$ = Opg au nè jour après le traitement ;

T2 = Opg initial du lot traité ;
$\mathrm{C} 1=\mathrm{Opg}$ au nè jour après le traitement du lot témoin ;

C2 = Opg initial du lot témoin.

A l'échelle d'un lot, le calcul du taux global de réduction de l'Opg a été basé sur la moyenne arithmétique des Opg individuels ainsi que sur la moyenne des pourcentages de réduction d'excrétion fécale individuels.

Nous avons considéré les valeurs d'efficacité négatives $(-\mathrm{X} \%)$ comme équivalent à zéro.

La première récolte de crottins a été effectuée à $\mathrm{J} 0$ et la seconde récolte à $\mathrm{J} 10$. Le délai de 10 à 11 jours est juste suffisant pour l'expulsion des vers tués et avant une réinfestation de l'animal.

Les calculs statistiques (comparaison de moyennes et de pourcentages) ont été effectués à l'aide du logiciel Microsoft Excel (V.2000), le programme FECRT et le logiciel Statistica (V.97, StatSoft, Tulsa, OK 74104, USA), avec un seuil de signification de $\mathrm{p}<0,05$.

\section{RESULTATS}

Les Tableaux 1 et 2 résument l'essentiel des nos résultats. D'après ces tableaux, le taux d'infestation des animaux par les strongles digestifs est similaire lors de deux prélèvements. Du Tableau 1, il ressort que le pourcentage de réduction de l'excrétion fécale des œufs après différents traitements a été nul chez les animaux témoins non traités, faible chez ceux traités aux benzimidazoles $(29,9$ à 31,5$)$ et élevé chez les animaux traités à l'ivermectine $(95,6)$. Le Tableau 2 montre qu'après administration des traitements aux différents groupes d'animaux, le pourcentage de réduction de l'excrétion fécale des œufs a été plus élevé chez les animaux traités à l'ivermectine $(96,7)$, presque nul $(0,7) \mathrm{chez}$ les témoins non traités et faible $(43,9-48,9)$ chez ceux traités aux benzimidazoles.

Le taux de réduction de l'excrétion fécale des œufs est plus élevé avec l'ivermectine par rapport aux deux benzimidazoles utilisés lors de cette étude. 
Tableau 1 : Fréquence et taux de réduction des strongles après divers traitements en 2012.

\begin{tabular}{lccc}
\hline Traitements $(\mathbf{n = 1 6})$ & $\mathbf{J 0}$ & $\mathbf{J 1 0}$ & \% de réduction \\
\hline Témoins non traités & $537,4 \pm 23,6$ & $704,3 \pm 17,8$ & 0 \\
Traités à l'albendazole & $548,6 \pm 39,5$ & $286,8 \pm 14,2$ & 31,5 \\
Traités à l'oxfendazole & $612,3 \pm 21,8$ & $327,2 \pm 21,9$ & 29,9 \\
Traités à l'ivermectine & $494,5 \pm 16,8$ & $16,6 \pm 5,2$ & 95,6 \\
\hline
\end{tabular}

Tableau 2 : Fréquence et taux de réduction des strongles après divers traitements en 2013.

\begin{tabular}{lccc}
\hline Traitements $(\mathbf{n}=\mathbf{1 5})$ & $\mathbf{J 0}$ & $\mathbf{J 1 0}$ & \% de réduction \\
\hline Témoins non traités & $617,8 \pm 28,5$ & $613,7 \pm 22,9$ & 0,7 \\
Traités à l'albendazole & $586,3 \pm 48,2$ & $301,9 \pm 17,6$ & 48,9 \\
Traités à l'oxfendazole & $573,6 \pm 31,5$ & $321,6 \pm 19,2$ & 43,9 \\
Traités à l'ivermectine & $603,5 \pm 39,4$ & $19,9 \pm 4,7$ & 96,7 \\
\hline
\end{tabular}

\section{DISCUSSION}

Des études rapportent de plus en plus des cas de résistances aux antihelminthiques (Waller, 1997 ; Kaplan, 2004 ; Torres-Acosta et al., 2012). Mais l'ampleur, les facteurs favorisants et le mécanisme de ces résistances font encore largement polémique (Okombe, 2011).

Nous avons considéré dans cette étude, qu'une résistance est présente au sein d'un troupeau, quand l'efficacité du traitement anthelminthique considéré induit une réduction de l'excrétion fécale d'œufs du parasite inférieure à $90 \%$, et ce alors que le traitement est correctement effectué.

Les échecs de vermifugation chez les caprins sont de plus en plus des phénomènes affectant les élevages dans les milieux tropicaux. Ces échecs peuvent relever de deux phénomènes distincts: d'une part, d'une inefficacité des anthelminthiques aux doses préconisées et d'autre part, d'une résistance vraie des populations parasitaires.

De nombreux travaux s'appuyant sur des tests in vitro (tests d'éclosion des œufs ou de paralysie des larves), ont démontré l'existence des souches résistantes de strongles digestifs chez les caprins (Geerts et al., 1990 ; Kerboeuf and Hubert, 1990 ; Borgsteede et al., 2007).
Dans notre étude, un phénomène de résistance pourrait être suspecté. En effet, dans les conditions décrites précédemment, trois anthelminthiques parmi les plus utilisés dans la région, à savoir l'ivermectine, l'oxfendazole et l'albendazole, ont été administrés à nos animaux d'étude dans un respect strict des conditions de vermifugation, particulièrement de la posologie caprine.

Le FECRT réalisé sur ces caprins fait suspecter l'existence des souches de nématodes résistantes aux benzimidazoles étudiés (albendazole et oxfendazole). Bien que certains auteurs indiquent des limites différentes en fonction de l'anthelminthique utilisé, par exemple: $95 \%$ pour le fenbendazole et l'ivermectine; $90 \%$ pour le Pyrantel (Pook et al., 2002), nous avons retenu le seuil, moins rigoureux, de $90 \%$.

Ainsi, un taux de réduction fécale inférieur à $90 \%$ indique une résistance des parasites au produit (Coles et al., 1992).

Ce taux de réduction fécale dans notre étude ne dépasse pas en moyenne $50 \%$ pour les benzimidazoles. Par contre, l'ivermectine a montré une bonne efficacité (le taux de réduction fécale dépasse la moyenne de $95 \%$ quelle que soit la ferme et au cours de deux traitements). Ce résultat est comparable à celui obtenu en Algérie (Boulkaboul et al., 
2006 ; 2010). Le test FECRT a révélé une différence significative $(\mathrm{p}<0,05)$ de l'efficacité de l'ivermectine par rapport à l'albendazole et à l'oxfendazole et cela dans les deux fermes et au cours de deux moments de l'étude.

En effet, certains éléments peuvent être retenus dans l'explication de cette suspicion.

Le premier est le manque d'alternance dans l'utilisation des anthelminthiques dans les exploitations concernées. Les benzimidazoles, à cause probablement de leur prix d'achat et de leur disponibilité sur le marché local, sont les seuls à être utilisés contre les strongles gastro-intestinaux avec comme anthelminthique de choix l'albendazole (92\%). L'usage exclusif d'un seul anthelminthique favoriserait la pression de sélection sur les parasites (Boulkaboul et al., 2006). En effet, l'usage alterné d'anthelminthiques de différents groupes pharmacologiques et présentant un mécanisme d'action différent ralentit le développement de résistance (Coles et al., 1992). L'apparition de résistance vis-à-vis d'une substance donnée compromet aussi l'action d'autres substances du même groupe pharmacologique et possédant un même mécanisme d'action (Okombe, 2011). Tous les benzimidazoles ayant le même mode d'action, les mécanismes de résistance sont identiques pour toutes les molécules de cette famille. C'est ce qui pourrait expliquer le comportement pharmacologique observé sur les parasites tant avec l'albendazole qu'avec l'oxfendazole.

A ce manque d'alternance, il ya lieu d'évoquer l'influence des traitements. En effet, la relation fréquence des traitements développement de la résistance a ainsi plusieurs fois été démontrée et n'est pas à écarter totalement des hypothèses pouvant expliquer cette suspicion de la résistance (Boulkaboul et al., 2006). L'apparition de la résistance est aussi proportionnelle au nombre d'interventions annuelles. Elle est très élevée en cas des traitements mensuels (De Graef et al., 2013). C'est celui-là d'ailleurs le cas dans ces deux fermes où les animaux reçoivent un traitement anthelminthique tous les deux mois, soit 6 fois par an. En effet, un nombre élevé de traitement conduit à une surprotection qui entrave la constitution d'une immunité permettant de résister aux infections naturelles. Ces traitements ne se contentent pas de prévenir les contaminations parasitaires, mais peuvent aussi diminuer le développement immunitaire contre ces mêmes parasites (Kerboeuf and Hubert, 1990).

L'autre élément est relatif au respect de la posologie. Le sous-dosage d'anthelminthiques, surtout lié à la sousestimation du poids corporel, est l'un des facteurs contribuant à l'apparition de résistance. Les sous-dosages apparaissent fréquemment chez les chèvres en raison de l'extrapolation aux chèvres des doses destinées aux moutons, alors que les substances actives du médicament présentent une biodisponibilité plus basse et une action plus faible chez la chèvre. Les doses des benzimidazoles devraient être 2 fois plus élevées chez les chèvres (Chartier et al., 1996 ; Chartier et Hoste, 1997). Sur le terrain, les traitements anthelminthiques sont généralement des traitements de masse réalisés dans $90 \%$ des cas par les éleveurs. Quand les traitements sont réalisés par les vétérinaires, la plupart de ceux-ci se basent sur une estimation visuelle du poids des animaux pour calculer la dose à administrer. Zouiten (2006) démontre que ce genre d'estimation aboutit, dans $75 \%$ des cas, à une administration d'une posologie qui s'éloigne de celle recommandée par le fabricant. Ceci a été remarqué surtout pour l'albendazole qui reste l'anthelminthique le plus utilisé. Par contre, les vétérinaires sont plus vigilants concernant le lévamisole injectable à cause probablement des effets toxiques chez l'animal suite à un surdosage.

\section{Conclusion}

Au terme de cette étude, nous pouvons conclure que la résistance aux benzimidazoles 
est fortement suspectée dans les deux fermes où s'est déroulée notre étude. Ce soupçon est d'autant plus fondé que les traitements sont exécutés en majeur partie par les éleveurs d'une manière anarchique et aléatoire (non respect de la fréquence de traitement et de la posologie, absence totale de rotation des molécules, utilisation abusive d'un seul produit). L'albendazole et l'oxfendazole ont montré un faible taux de réduction fécale durant les deux moments de notre étude, par comparaison à l'ivermectine.

Ces premiers résultats confirment ceux rapportés dans la littérature concernant le développement d'une résistance dans la famille des benzimidazoles. Le danger est alors réel.

Ces résultats sont une base pour les recherches ultérieures à mener dans le but de confirmer cette résistance par d'autres tests dans des conditions d'étude contrôlées intégrant notamment des infestations artificielles et des suivis plus ciblés, de manière à écarter les fausses résistances dues à des problèmes techniques.

\section{REMERCIEMENTS}

Les auteurs remercient les Docteurs vétérinaires Francis FEKOU DJOUKEN et Gaël NZUZI pour leur collaboration.

\section{REFERENCES}

Ba H, Geerts S. 1998. La résistance aux benzimidazoles des nématodes gastrointestinaux des petits ruminants en Gambie et au Sénégal. Revue Elevage et Médecine Vétérinaire des Pays Tropicaux, 51(3): 207-210.

Besier RB, Love SCJ. 2003. Anthelmintic resistance in sheep nematodes in Australia: the need for new approaches. Australian Journal of Experimental Agriculture, 43(12): 1383 - 1391.

Borgsteede FH, Dercksen DD, Huijbers R. 2007. Doramectin and albendazole resistance in sheep in the Netherlands. Veterinary Parasitology, 144, 180-183.
Boulkaboul A, Bouakkaz A, Kerboeuf D. 2006. Détection d'une résistance aux benzimidazoles chez les strongles digestifs du cheval en Algérie. Revue Méd. Vét., 157(2): 59-64.

Boulkaboul A, Boucif A, Senouci A. 2010. Recherche de la résistance des strongles aux anthelminthiques chez le mouton en Algérie. Revue Elevage et Médecine Vétérinaire des Pays Tropicaux, 63(3-4): 71-75.

Chartier C, Kulo A, Cabaret J. 1996. Absorption et métabolisme des benzimidazoles chez les caprins : relation avec l'efficacité contre Muellerius capillaris et les strongles gastrointestinaux. Revue Méd. Vét., 147(7): 531538.

Chartier C, Pors I, Hubert J, Rocheteau D, Benoit C, Bernard N. 1997. Prevalence of anthelmintic resistant nematodes in sheep and goats in Western France. Small Ruminant Research, 1615: 1-9.

Chartier C, Hoste, H. 1997. La thérapeutique anthelminthique chez les caprins. Le Point Vétérinaire, 28: 125-132.

Coles GC, Bauer C, Borgsteede FHM, Geerts S, Klei TR, Waller PJ. 1992. Word Association for the advancement of veterinary parasitology (W.A.A.V.P.) methods for the detection of anthemintic resistance in nematodes of veterinary importance. Vet. Parasitol., 44: 35- 44.

Coles GC, Jackson F, Pomroy WE, Prichard RK, von Samson-Himmelstjerna G, Silvestre A, Taylor MA, Vercruysse J. 2006. The detection of anthelmintic resistance in nematodes of veterinary importance. Veterinary Parasitology, 136, 167-185.

Condor GA, Campbell WC. 1995. Chemotherapy of nematode infections of veterinary importance with special reference to drug resistance. $A d v$. Parasitol., 35: 1-84.

Dash K, Hall K, Barger IA. 1988. The role of arithmetic and geometric worm egg counts in faecal egg count reduction test and in 
monitoring strategic drenching programs in sheep. Australian Veterinary Journal, 65: $66-68$.

De Graef J, Claerebout E, Geldhof P. 2013. Anthelmintic resistance of gastrointestinal cattle nematodes. Vlaams Diergeneeskundig Tijdschrift, 82: 113123.

Demeler J, Van Zeveren AM, Kleinschmidt N, Vercruysse J, Hoglund J, Koopmann R, Cabaret J, Claerebout E, Areskog M, von Samson-Himmelstjerna G. 2009. Monitoring the efficacy of ivermectin and albendazole against gastro intestinal nematodes of cattle in Northern Europe. Veterinary Parasitology, 160, 109-115.

FAO. 2004. Guidelines Resistance Management and Integrated Parasite Control in Ruminants. Animal Production and Health Division Agriculture Departement. Food and Agriculture Organisation on The United Nations: Rome; 78-118.

Geerts S, Bertels G, Bali SB, Brandt J, Kumar V. 1990. Benzimidazole resistance in nematodes on a dairy goat farm in Belgium. Vlaams Diergeneeskungig Tijdschrift, 59: 90-92.

Kaplan RM. 2004. Drug resistance in nematodes of veterinary importance: a status report. Trends in Parasitology, 20(10): 477-481.

Kerboeuf D, Hubert J. 1990. Efficacité comparée des famillles d'anthelminthiques vis-à-vis de strongles résistants aux benzimidazoles. Rec Méd. Vét., 166: 771775 .

Levecke B, Dobson RJ, Speybroeck N, Vercruysse J, Charlier J. 2012. Novel insights in the faecal egg count reduction test for monitoring drug effi acy against gastrointestinal nematodes of veterinary importance. Veterinary Parasitology, 188, 391-396.
Mukaratirwa S, Charakupa R, Hove T. 1997. A survey of anthelmintic resistance on ten sheep farm in Mashonaland East Province, Zimbabwe Tydskr. S.Afr.Vet.Ver., 68(4): 140-143.

Okombe EV, Mbumba T, Pongombo CS. 2013. Efficacité antiparasitaire de la poudre de graines de courge (Cucurbita moschata L.) sur les helminthes gastrointestinaux de la chèvre locale élevée à Lubumbashi en République Démocratique du Congo. Int. J. Biol. Chem. Sci., 7(3): 953-960.

Okombe EV. 2011. Activité antihelminthique de la poudre d'écorce de racine de Vitex thomasii De Wild (Verbenaceae) sur Haemonchus contortus chez la chèvre. Thèse, Université de Lubumbashi, Lubumbashi, p. 242.

Pook JF, Power ML, Sangster NC, Hodgson JL, Hodgson DR. 2002. Evaluation of test for anthelmintic resistance in cyathostomes. Vet. Parasitol., 106: 331343.

Suarez VH, Cristel SL. 2007. Anthelmintic resistance in cattle nematode in the western Pampeana Region of Argentina. Veterinary Parasitology, 144: 111-117.

Sutherland IA, Leathwick DM. 2010. Anthelmintic resistance in nematode parasites of cattle: a global issue? Trends in Parasitology, 27(4): 176-181.

Thienpont D, Rochette F, Vampari JS. 1979. Diagnostic de Verminose par Examen Coprologique. Janssen Research Foundation : Beerse.

Torres-Acosta JF, Mendoza-de-Gives P, Aguilar-Caballero AJ, Cuellar-Ordaz JA. 2012. Anthelmintic resistance in sheep farms: Update of the situation in the American continent. Veterinary Parasitology, 189: 89-96.

Urquhart GM, Amour J, Duncan JL, Dunn AA, Jennings FW. 1996. Veterinary 
Parasitology (2nd edn). Blackwell Science Ltd : United Kingdom.

Van Wyk JA, Malan FS, Randles JL. 1997. How long before resistance makes it impossible to control some field strains of Haemonchus contortus in South Africa with any of the modern anthelmintics. Vet. Parasitol., 70: 111-122.

Van Wyk JA, Stenson MO, Van Der Merwe JS, Vorster RJ, Viljoen PG. 1999. Anthelmintic resistance in South Africa: Surveys indicate an extremely serious situation in sheep and goat farming. Onderstepoort Journal of Veterinary Research, 66: 273-284.

Waller P. 1997. Anthelmintic resistance. Veterinary Parasitology, 72: 391-412.
Waruiru RM, Kogi JK, Weda EH, Ngotho JW. 1998a. Multiple resistance on a goat farm in Kenya. Veterinary Parasitology, 75: 191-197.

Waruiru RM, Ngotho JW, Mukuri JG. 1998b. Multiple and multigeneric anthelmintic resistance on a sheep farm in Kenya. Tropical Animal Health and Production, 30: $159-156$.

Zouiten H. 2006. Résistance aux anthelminthiques des nématodes parasites du tube digestif chez les ovins et les équidés au Maroc. Thèse, Université MohammedV - Agdal, Rabat, p. 138. 